\title{
Unresectable bulky chest wall recurrent breast cancer controlled with CT-guided interstitial high- dose-rate brachytherapy and external beam radiotherapy with adjuvant hormonal therapy - case report
}

\author{
Hiroaki Kunogi, MD', I-Chow Hsu, MD², Keisuke Sasai, MD' \\ 'Department of Radiation Oncology, Juntendo University, Tokyo, Japan, ${ }^{2}$ Department of Radiation Oncology, University of California \\ San Francisco, San Francisco, CA, USA
}

\begin{abstract}
Purpose: Bulky chest wall recurrence after mastectomy presents a therapeutic challenge because of high-dose of radiation required to control the disease, and its proximity to low-tolerance organs at risk. We report a case of successful computed tomography (CT)-guided high-dose-rate (HDR) salvage interstitial brachytherapy (ISBT) boost.

Material and methods: A 70-year-old female initially presented with a tumor in right breast, and was treated with mastectomy and adjuvant chemotherapy, followed by hormonal therapy for 5 years without adjuvant radiotherapy. In 2018, 20 years after the initial treatment, she developed unresectable chest wall recurrence that measured $10.5 \mathrm{~cm}$ $\times 7.3 \mathrm{~cm} \times 4.5 \mathrm{~cm}$, with bone and parietal pleura invasion. Biopsy revealed invasive pleomorphic lobular carcinoma [estrogen receptor (ER)-positive, progesterone receptor (PR)-negative, HER2-negative]. There was no evidence of metastatic disease.

Results: The patient underwent external beam radiotherapy (EBRT) plus ISBT. After EBRT of 50 Gy in 25 fractions was completed, CT-guided ISBT was performed as an outpatient treatment. HDR dose was 16 Gy delivered in 2 fractions with 2 implants. Dose was prescribed to gross tumor volume. ISBT plans were created using inverse planning simulated annealing (IPSA) algorithm. Gross tumor volume $\mathrm{D}_{90 \%}$ plus EBRT dose was 82 Gy equivalent dose of 2 Gy $\left(\mathrm{EQD}_{2}\right)$, assuming $\alpha / \beta$ of 4 for breast carcinoma. The patient continued on hormonal therapy. At the 30-month follow-up, the patient remains in remission. The tumor could not be detected by magnetic resonance imaging (MRI) or positron emission tomography (PET). There were no severe treatment-related complications.

Conclusions: CT-guided HDR ISBT boost can be a useful modality in individualizing treatment strategies for breast cancer patients with unresectable bulky chest wall recurrence.

Key words: breast cancer, interstitial brachytherapy, unresectable recurrent cancer.

\section{Purpose}

In the management of bulky chest wall recurrence of breast cancer, where there is no standard or clearly superior therapy, it is important to individualize patient care and treatment strategies [1]. Surgical resection of loco-regional recurrence should be expected if it may be safely administered with adjuvant systemic chemotherapy or hormonal therapy. Radiation therapy is also used to treat loco-regional recurrence in breast cancer patients after mastectomy [1, 2], especially in patients with unresectable tumors. In breast cancer patients with local recurrence, who have a long disease-free interval after mastectomy, long-term survival is often expected [3, 4]. In such cases, loco-regional salvage therapy should be administered with a curative intent.

Breast interstitial brachytherapy (ISBT) can be used as a boost after external beam radiotherapy (EBRT) or as monotherapy. A few studies have evaluated breast ISBT. Syed et al. reported that ISBT plus EBRT without surgery provides effective loco-regional control as an initial treatment in patients with locally advanced breast cancer [5]. Wu et al. demonstrated a re-irradiated case of high-doserate (HDR) ISBT alone for a bulky chest wall recurrence 
of breast cancer [6]. Roddiger et al. reported on 53 patients treated with HDR ISBT as neoadjuvant radiotherapy [7]. However, little is known about the clinical value of computed tomography (CT)-guided breast ISBT, especially for breast cancer patients with unresectable bulky tumors, even though CT-guided ISBT is a safe and feasible treatment approach for many cancer types [8-10]. Here, we describe the technique of CT-guided HDR ISBT and the efficacy of ISBT plus EBRT with hormonal therapy as a therapeutic option in patients with unresectable chest wall recurrence of breast cancer.

\section{Case report}

\section{Patient presentation}

We report the case of a 70-year-old woman who underwent HDR ISBT combined with EBRT as a radical treatment for unresectable bulky chest wall recurrence of breast cancer with bone and parietal pleura invasion. The patient was firstly diagnosed with a tumor in the right breast in 1998. The time elapsed from initial treatment was very long (20 years) and information about the initial histopathology or tumor stage was unavailable. She underwent a total mastectomy with adjuvant chemotherapy, followed by hormonal therapy for 5 years, with no adjuvant radiotherapy given. In 2008, 10 years after total mastectomy, follow-up confirmed the absence of recurrent disease. In 2018, the patient reported pain and swelling near the surgical scar and visited the hospital for a check-up. Ultrasound (US) revealed a bulky tumor; fine-needle biopsy confirmed the presence of malignant cells. Pathological evaluation indicated invasive pleomorphic lobular carcinoma positive for estrogen receptor (ER), and negative for progesterone receptor (PR) and HER2. This patient was clinically suspected to have recurrent breast cancer based on the tumor location. Ki-67 index was $22 \%$, and ${ }^{18} \mathrm{~F}$-labeled fluoro-2-deoxyglucose $\left({ }^{18} \mathrm{~F}-\mathrm{FDG}\right)$ positron emission tomography (PET) with CT

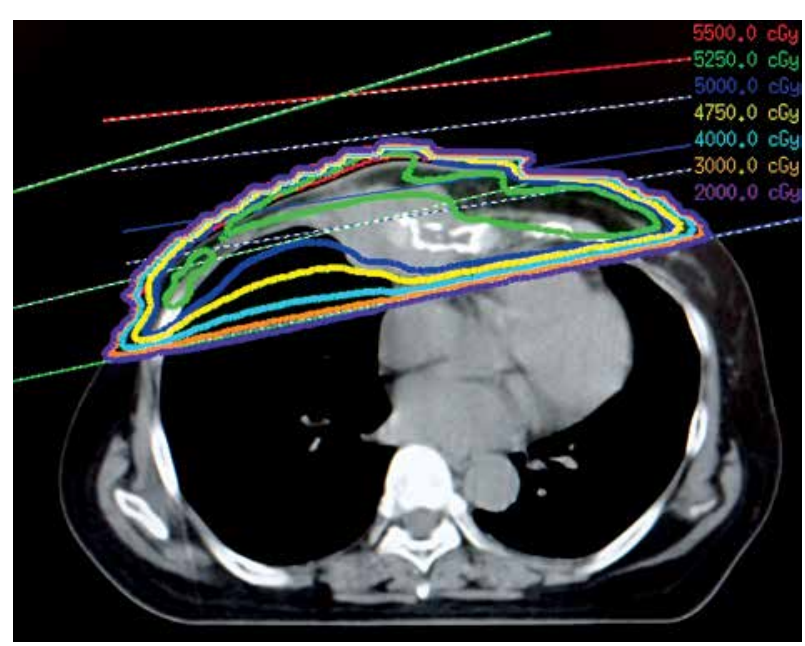

Fig. 1. Dose distribution in external beam radiotherapy (EBRT) planning. In this EBRT plan, the lung $\mathrm{V}_{5 \mathrm{~Gy}}$ and $\mathrm{V}_{\text {20Gy }}$ (i.e., the percent volumes of both lungs receiving 5 Gy and 20 Gy, respectively) were $20 \%$ and $13 \%$, respectively, and the mean heart dose was $5.6 \mathrm{~Gy}$ showed no lymph node or distant metastases (rT4N0M0). Plain and dynamic magnetic resonance imaging (MRI) was also performed to evaluate the local lesion. There were no tumors in the left breast on mammography, US, and dynamic MRI. Radiotherapy and hormonal therapy were recommended for the chest wall recurrence, which was approximately $10.5 \mathrm{~cm} \times 7.3 \mathrm{~cm} \times 4.5 \mathrm{~cm}$, including erythematous skin involvement (approx. $1 \mathrm{~cm}$ ). Surgery was not recommended due to the bone and pleura invasion. Written informed consent for EBRT plus HDR brachytherapy as curative radiotherapy was obtained.

\section{Treatment}

Hormonal therapy with anastrozole was initiated, followed by EBRT. During EBRT, the gross tumor volume (GTV) was contoured. No margin was added to the GTV to create clinical target volume (CTV). The CTV was expanded by $5 \mathrm{~mm}$ for planning target volume (PTV) as a margin to compensate for uncertainties in treatment delivery. During EBRT, 50 Gy in 25 fractions was delivered to the PTV using 3D conformal radiotherapy (Figure 1). The lungs and heart dosimetric parameters are described in Table 1 . The patient was treated with $10-\mathrm{MV}$ photons with a $5 \mathrm{~mm}$ bolus using an Elekta Synergy linear accelerator (Elekta; Stockholm, Sweden). ISBT was performed after completion of EBRT.

Before the first implantation, repeated CT revealed a slight reduction in the tumor volume $(9.0 \mathrm{~cm} \times 6.5 \mathrm{~cm}$ $\times 3.5 \mathrm{~cm}$ ). The first implant was performed 1 week after EBRT, and the second implant was done 4 weeks after the first implant. There was no evidence of lung invasion

Table 1. Dose-volume histograms of the gross tumor volume (GTV), lungs, and heart during external beam radiotherapy (EBRT) and the first and second interstitial brachytherapy (ISBT) boost

\begin{tabular}{lccc} 
& EBRT 50 Gy & $\begin{array}{c}\text { First ISBT } \\
\text { boost }\end{array}$ & $\begin{array}{c}\text { Second ISBT } \\
\text { boost }\end{array}$ \\
\hline GTV & & 8.1 & 8.0 \\
\hline$D_{90 \%}(G y)$ & - & 91.0 & 90.0 \\
\hline$V_{100 \%}(\%)$ & - & 60.0 & 55.0 \\
\hline$V_{150 \%}(\%)$ & - & 38.0 & 34.0 \\
\hline$V_{200 \%}(\%)$ & - & & \\
\hline Lungs & & 1.0 & 0.7 \\
\hline$V_{5 G y}(\%)$ & 20.5 & 0.0 & 0.0 \\
\hline$V_{10 G y}(\%)$ & 15.8 & 0.0 & 0.0 \\
\hline$V_{15 G y}(\%)$ & 13.9 & 0.0 & 0.0 \\
\hline$V_{20 G y}(\%)$ & 12.8 & & \\
\hline Heart & & 2.6 & 0.5 \\
\hline$V_{5 G y}(\%)$ & 17.3 & 0.0 & 0.0 \\
\hline$V_{10 G y}(\%)$ & 10.9 & 0.0 & 0.0 \\
\hline$V_{20 G y}(\%)$ & 7.3 & 0.0 & 0.0 \\
\hline$V_{30 G y}(\%)$ & 4.9 & 2.7 & 2.2 \\
\hline Mean $(G y)$ & 5.6 & &
\end{tabular}





Fig. 2. Implantation of brachytherapy flexible catheters (A) and flexible catheters fixed with dental putty (light blue) (B). The putty was sutured to the skin using a thread (white arrow) to avoid catheter's displacement. Catheters were attached to the putty using instant glue (tip of black arrow) to strengthen the fixation further

because the lung did not move with the tumor on US imaging under natural respiration. Before implantation, the insertion points and catheters' paths were determined based on CT. To minimize the area of skin affected by high-dose, in the second implant, we selected different insertion points and catheters' paths from those used in the first implant. The catheters were implanted under local anesthesia and after the administration of intravenous non-steroidal anti-inflammatory drugs in our outpatient clinic. During catheter implantation, the patient was placed in a supine position in an HDR room with a dedicated CT system (Figure 2A). Flexi-guide catheters (outer diameter, 5 French; length, $24 \mathrm{~cm}$ ) were used as brachytherapy catheters. Sixteen-gauge needles were used to puncture the skin. The needles were replaced with the flexi-guide catheters after skin puncture. This process was repeated for each flexi-guide catheter. After penetrating the skin, the flexi-guide catheters were advanced using free-hand technique under serial CT imaging. We advanced each flexi-guide catheter carefully and slowly from the skin, lateral medially across the lesion, and toward anterior portion of the sternum under CT guidance to avoid puncturing the blood vessels and lungs. CT guidance provides good bone and soft tissue visualization. Therefore, CT enabled the brachytherapist to visualize the entire tumor with sternum invasion, and the catheters were placed just anterior of the sternum (Figure 3). The CT dose index and dose-length product from CT im-
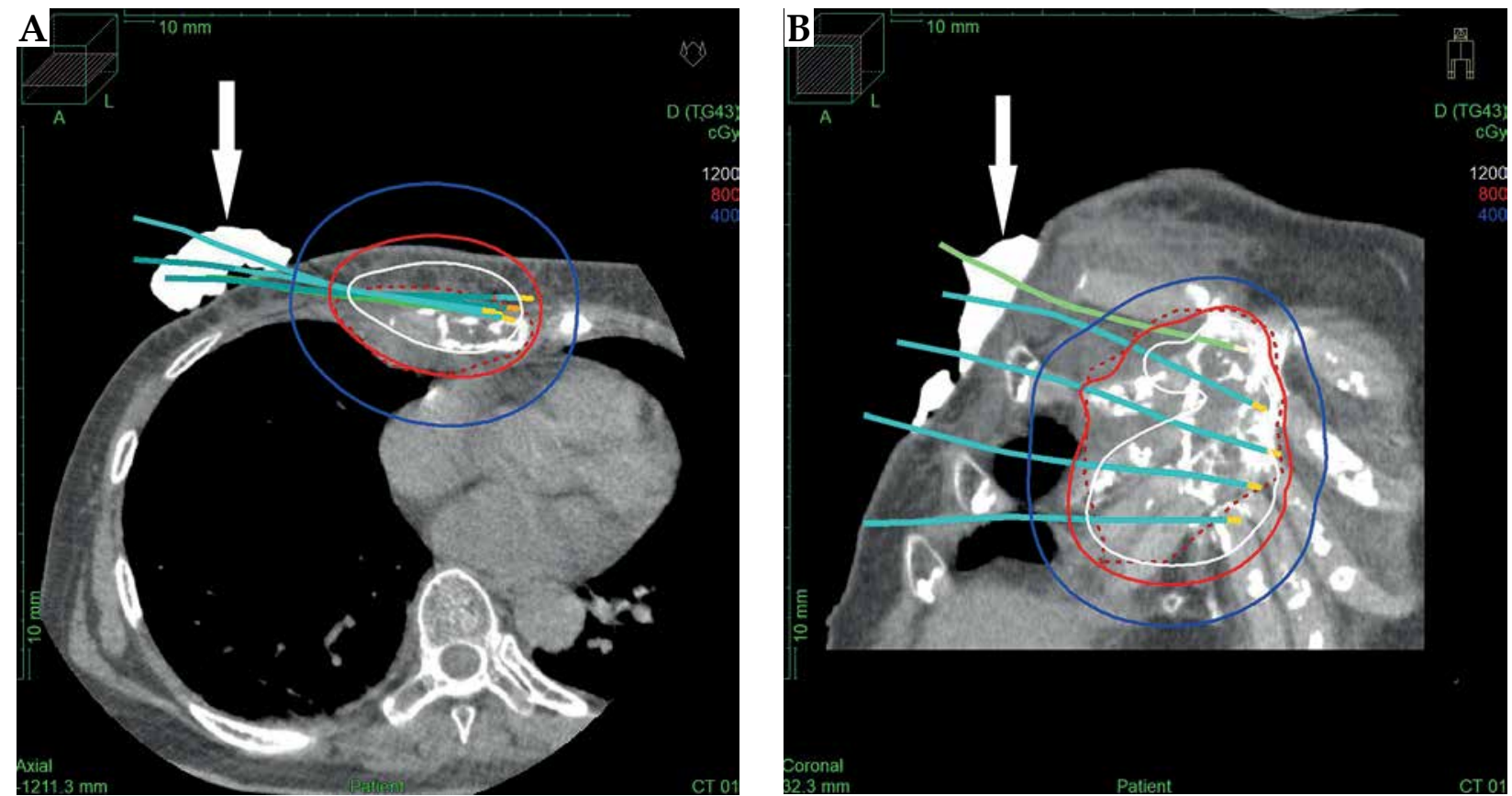

Fig. 3. Axial (A) and coronal (B) CT images of the gross tumor volume (GTV; red dashed lines), including the sternum lesions receiving 4 Gy (blue lines), 8 Gy (red lines), and $12 \mathrm{~Gy}$ (white lines). The catheters were implanted, penetrating the tumor and reaching the front of the sternum. The dental putty can also be seen (white arrows) 
aging for the implants were $475 \mathrm{mGy}$ and $7081 \mathrm{mGy}$ * $\mathrm{cm}$, respectively. Once the catheter was properly inserted, the inner obturators were removed while maintaining the position of flexible catheters. The catheters were secured using dental putty (Exafine putty type ${ }^{\circledR}$; GC Corporation, Tokyo, Japan). We used a similar catheter fixation technique for prostate brachytherapy [11]. After the dental putty hardened, it was sutured to the chest skin to hold the catheters in place (Figure 2B). Instant glue was applied to the catheters to secure them to the putty.

Brachytherapy planning CT was performed with a $1 \mathrm{~mm}$ slice thickness, and the GTV was contoured. Organs at risk (OARs) included the skin, ribs, lung, and heart. The prescription dose was set to $8 \mathrm{~Gy}$ per fraction. Three-dimensional brachytherapy plans were generated using Elekta Nucletron Oncentra treatment planning system version 4.5 , with the inverse planning simulated annealing (IPSA) algorithm. Total equivalent dose of 2 Gy $\left(\mathrm{EQD}_{2}\right)$ was calculated based on the assumption an $\alpha / \beta$ of 4 for breast carcinoma [12]. IPSA was used to achieve optimal dose distribution to the GTV $\mathrm{D}_{90 \%}$ (i.e., minimum dose delivered to $90 \%$ of the GTV). The GTV was adjacent to the lung and heart due to the pleura invasion, so the prescription isodose line was intended to be positioned at the boundary between the GTV and the lung/heart. Lungs and heart dosimetric parameters in the first and second ISBT are described in Table 1 . Not all of the lungs were scanned in the ISBT planning CT, therefore dosimetric parameters of the lungs were calculated using the total lung volume in the EBRT planning CT. GTV coverage was prioritized over skin-sparing, while the irradiated skin volume was kept as low as possible. The total GTV $\mathrm{D}_{90 \%}$, including the EBRT dose, was $82 \mathrm{~Gy} \mathrm{EQD}_{2}$. Dose distribution is presented in Figure 3. CT was performed just before irradiation to confirm that the implanted catheters were not displaced. The patient was treated on the CT table without moving after the implantation. Two single-fraction implants of 8 Gy (interfractional interval of 28 days) were delivered to the GTV using MicroSelectron-HDR ${ }^{\circledR}$ (Nucletron B.V., Veenendaal, The Netherlands) with an ${ }^{192}$ Ir source. Five and seven catheters were used in the first and second ISBT, respectively. On the day of implantation, the patient received a single-fraction, after which the implant was removed. The patient was discharged after removing the catheters. No complications occurred during the whole brachytherapy procedure. After radiotherapy, hormonal therapy was administered until the last follow-up.
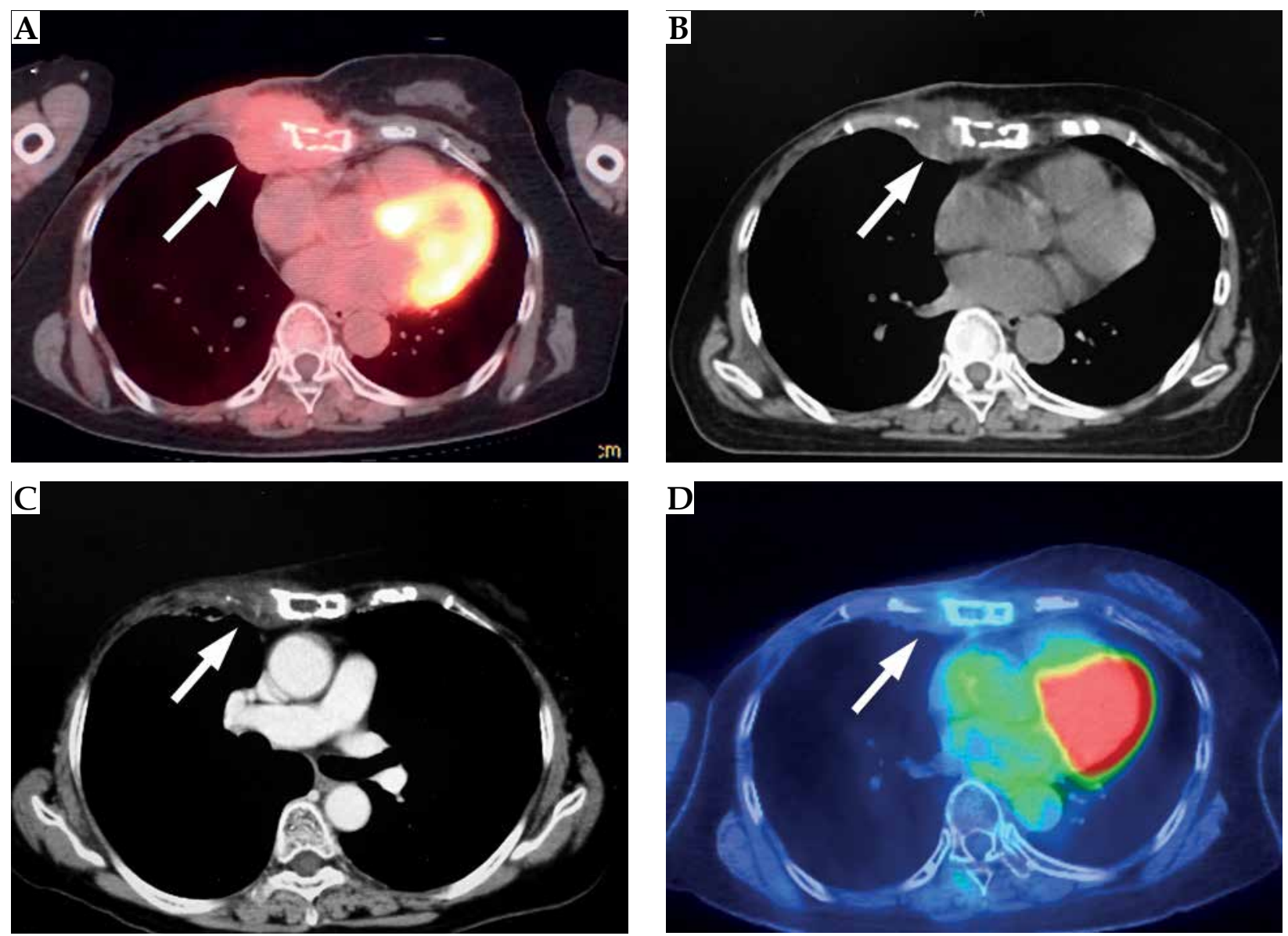

Fig. 4. Axial ${ }^{18}$ F-FDG-PET/CT image before radiotherapy (A), axial plain CT image before the second interstitial brachytherapy (ISBT) (B), enhanced axial CT image at 6 months (C), and axial ${ }^{18}$ F-FDG-PET/CT image at 26 months (D). The bulky tumor with bone invasion (A, white arrows) on the right chest wall decreased in size (B and $\mathbf{C}$, white arrows) and finally disappeared (D, white arrows) 
A blood tumor marker test was performed every month. Enhanced CT was done after 6 months, and ${ }^{18}$ F-FDG-PET/CT was performed after 14 and 26 months. Chest MRI was also completed after 28 months. Adverse events were evaluated using the common terminology criteria for adverse events version 4.0 (CTCAE v.4) [13]. Before and after salvage treatment, the levels of breast cancer antigen 225 (BCA225) and carbohydrate antigen 15-3 (CA15-3) were within normal physiological range. Serum level of the breast cancer marker carcinoembryonic antigen (CEA), which was $19.5 \mathrm{ng} / \mathrm{ml}$ pre-treatment, gradually decreased at 3 months after the first day of treatment; serum CEA levels remained within normal ranges through the last follow-up (CEA at the last follow-up of 30 months, $2.6 \mathrm{ng} / \mathrm{ml})$. Enhanced CT examination at 6 months demonstrated that the size of the tumor was considerably reduced (Figure 4). Furthermore, MRI at 28 months and ${ }^{18}$ F-FDG-PET/CT at 26 months indicated that the bulky tumor had disappeared (Figures 4 and 5). There was no evidence of tumor recurrence at the 30-month follow-up. The patient exhibited acute dermatitis (grade 2) in the irradiated area (EBRT and ISBT) for nearly 2 weeks after irradiation, which was managed with a steroid ointment. White petroleum (Vaseline) was used to moisturize the irradiated skin. Telangiectasia was apparent from 1 year after radiotherapy, until the last follow-up (Figure 6). CT and ${ }^{18} \mathrm{~F}-\mathrm{FDG}-\mathrm{PET} / \mathrm{CT}$ revealed a radiation-induced inflammatory change in the lungs (grade 1). There were no severe (grade 3 or higher) complications. The pre-treatment pain near the surgical scar disappeared after irradiation.

\section{Discussion}

Radiation oncologists are often asked to manage patients with localized, yet unresectable disease. Local control is essential for quality of life and survival of these patients. The optimal tumor control dose for unresectable bulky breast tumors and optimal irradiation method remain to be determined, although NCCN guidelines [2] state that radiotherapy may be considered for unresectable local recurrence in post-mastectomy patients with no prior radiotherapy. In the absence of established standard therapy, a radiation oncologist needs to individualize treatment and provide personalized care. Brachytherapy can provide the most conformal and precise radiotherapy. Based on the case described here, we believe that outpatient CT-guided ISBT boost is a beneficial therapeutic option for breast cancer patients with unresectable chest wall recurrence.
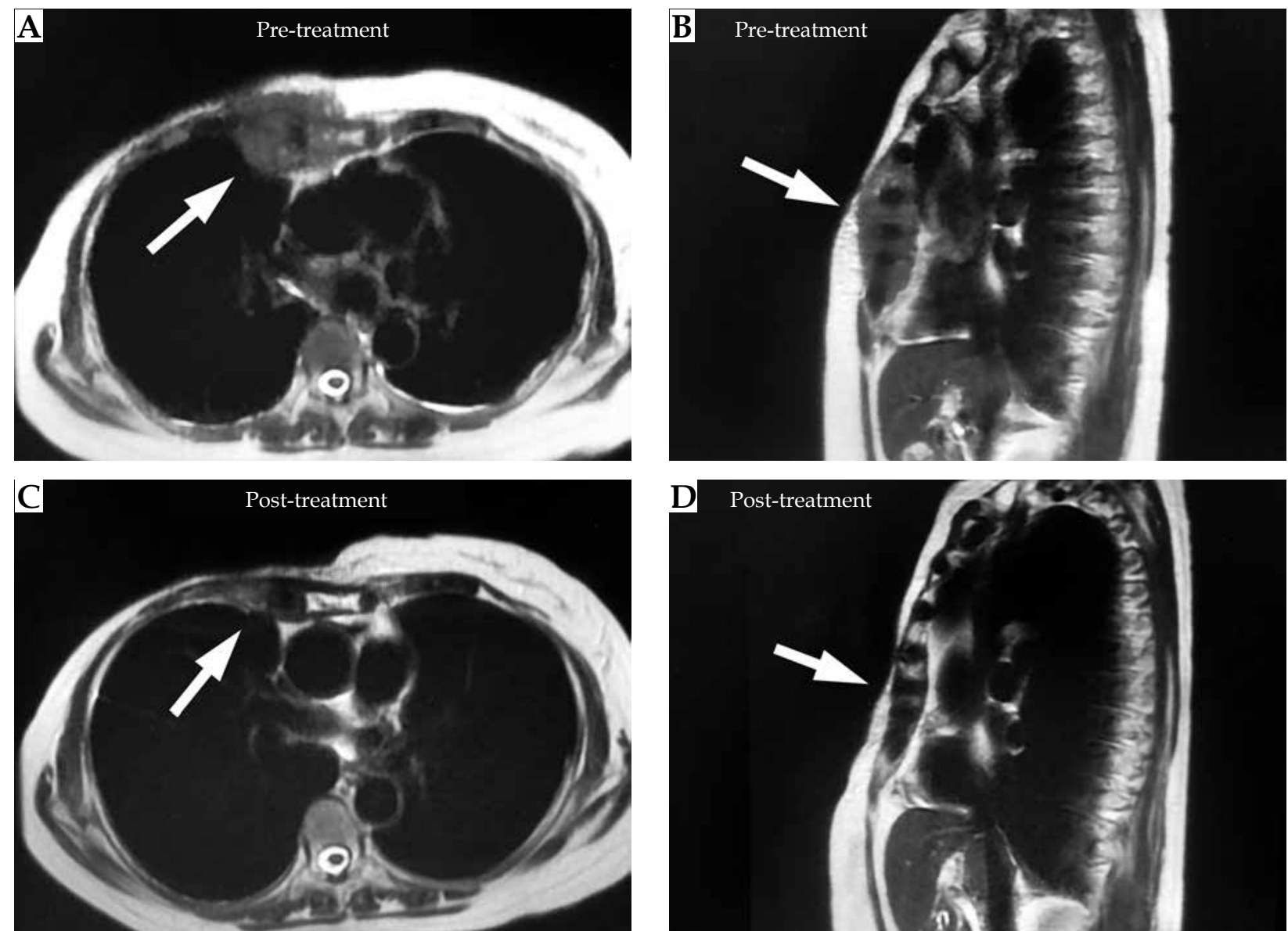

Fig. 5. Axial (A) and sagittal (B) T2-weighted MRI before radiotherapy. Axial (C) and sagittal (D) T2-weighted MRI at 28 months. The bulky tumor with bone invasion (A and $\mathbf{B}$, white arrows) on the right chest wall disappeared (C and $\mathbf{D}$, white arrows) 


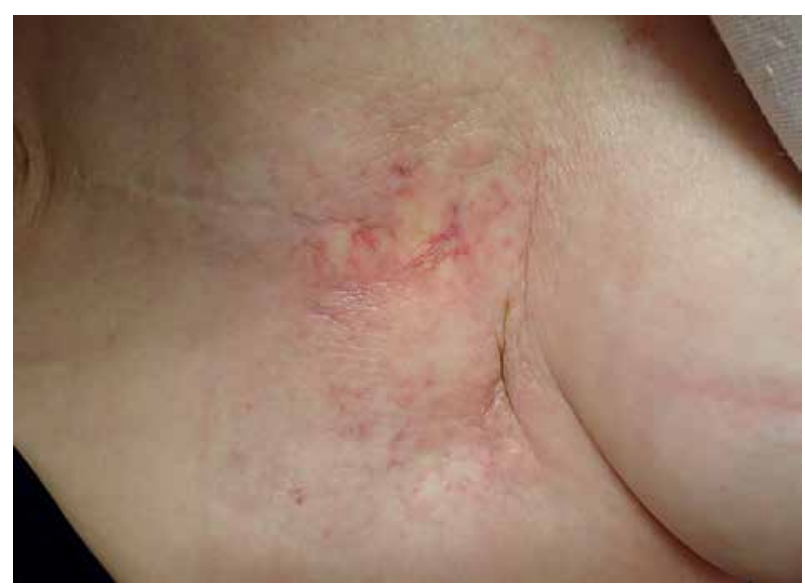

Fig. 6. The right chest wall at 30 months. Telangiectasia is apparent on the skin surface

We found CT guidance useful in this case. CT can visualize the entire tumor, including bone invasion. $\mathrm{Wu}$ et al. reported the use of US-guided HDR ISBT to treat a bulky chest wall recurrence in a breast cancer patient [6]. In our case, the additional information provided by CT was helpful in accurately placing the catheters. Therefore, if a resource is available, we believe this approach should be considered.

The application of brachytherapy may achieve improvement of loco-regional control of bulky unresectable breast tumor, as brachytherapy can deliver a higher internal dose, which may improve control of radio-resistant tumor cells and reduce the risk of viable residual disease. In patients with bulky unresectable tumors, EBRT alone may not provide adequate loco-regional control [14]. Fröhlich et al. [15] demonstrated that EBRT plus ISBT after breast surgery allowed for delivery of higher doses compared with EBRT alone.

Brachytherapy permits for precise dose delivery despite chest movements due to respiration. The implanted catheters can retain a stable position in relation to the tumor. Fixation of the catheters is essential to reduce the risk of catheter displacement. Here, we used dental putty to custom-fit patient's anatomy and the implanted catheters. Alternative method of implant using a template may be also feasible. However, we found free-hand method technically simple, with additional benefit of being very adaptable for a variety of different clinical presentations. Also, we accomplished our goal of securing the catheters with a custom-fitted mold, which we believe, further minimized the patient's discomfort. Furthermore, the implantation and treatment were performed in the same position without moving the patient, which further reduced the risk of catheter displacement. A HDR room with a dedicated CT system facilitated this approach.

The total number of catheters may depend on not only target size and shape, but also dose distribution from prior treatment fractions. In the outpatient setting, we used 5 catheters during the first ISBT, whereas more catheters were applied in the second ISBT to further improve dose distribution. Using more catheters provided a better dose distribution at the expense of increased op- erating time. However, more catheters may also cause more patient's discomfort. To further minimize hot spots in the treatment volume, we shifted catheters' positions between the first and the second ISBT by selecting different insertion points and catheters' paths to reduce higher dose volumes as much as possible. Fortunately, our patient did not suffer from severe skin toxicity associated with brachytherapy hot spots. In general, due to close proximity between treatment volume and the skin, it may be difficult to accomplish strict skin sparing without compromising tumor coverage. It is especially challenging if there is a gross tumor invasion of the skin. Application of stricter skin dose constraints may result in missing the target and local recurrence. More data is needed to resolve this issue. The optimal dose for treatment of bulky breast tumor recurrence has not been established. The total dose for the GTV $D_{90 \%}$ was intended to be $\geq 80$ Gy $\left(\mathrm{EQD}_{2}\right)$, and therefore, the total dose was $82 \mathrm{~Gy}$. Syed et al. reported results of 90-100 Gy using EBRT plus ISBT for T3 and T4 breast tumors [5], and we believe that this is a useful guideline until more data become available. Currently, there are few specific radiotherapeutic recommendations for unresectable loco-regional recurrence in breast cancer patients after mastectomy with no prior radiotherapy [2]. Neoadjuvant radiotherapy using HDR ISBT may be a treatment option for unresectable breast tumors. Roddiger et al. demonstrated the possibility of neoadjuvant HDR ISBT for breast cancer patients, who could not be treated with breast-conserving surgery due to the initial tumor size or unfavorable breast-tumor ratio [7]. Neoadjuvant radiotherapy may be a treatment option if it is not for a bone or parietal pleura invasion case.

It is always important to maximize loco-regional control and to minimize the treatment burden for our patients. Our treatment was delivered with EBRT and two outpatients' implants. It may be possible to deliver multiple fractions or whole treatment with a single-implant; however, we selected single-fraction implants to re-distribute high-dose volume by slightly varying catheters' positions with each implant. Moreover, two implants were chosen to deliver the treatment, a reasonable number for the patient in our outpatient setting. It may be possible to further reduce the treatment time by modifying the fractionation and contribution of the total dose by modality. Further investigation is warranted.

\section{Conclusions}

The application of CT-guided interstitial HDR brachytherapy boost combined with hormonal therapy may provide efficient loco-regional control in individualizing treatments' strategies for unresectable bulky chest wall recurrence of breast cancer.

\section{Disclosure}

The authors report no conflict of interest.

\section{References}

1. Halyard MY, Harris EE, Bailey L et al. ACR Appropriateness Criteria local-regional recurrence (LRR) and salvage 
surgery-breast cancer. Oncology (Williston Park) 2014; 28: 157-

$164, \mathrm{C} 3$.

2. https://www.nccn.org/professionals/physician_gls/pdf/ breast.pdf

3. Aebi S, Gelber S, Anderson SJ et al. Chemotherapy for isolated locoregional recurrence of breast cancer (CALOR): a randomised trial. Lancet Oncol 2014; 15: 156-163.

4. Kuo SH, Huang CS, Kuo WH et al. Comprehensive locoregional treatment and systemic therapy for postmastectomy isolated locoregional recurrence. Int J Radiat Oncol Biol Phys 2008; 72: 1456-1464.

5. Syed AM, Puthawala A, Fleming P et al. Combination of external and interstitial irradiation in the primary management of breast carcinoma. Cancer 1980; 46: 1360-1365.

6. Wu N, Chen Q, Zhao Z et al. Interstitial brachytherapy technique for chest wall refractory recurrence of breast cancer. J Contemp Brachytherapy 2015; 7: 290-294.

7. Roddiger SJ, Kolotas C, Filipowicz I et al. Neoadjuvant interstitial high-dose-rate (HDR) brachytherapy combined with systemic chemotherapy in patients with breast cancer. Strahlenther Onkol 2006; 182: 22-29.

8. Kishi K, Sonomura T, Shirai S et al. Reirradiation of paraaortic lymph node metastasis by brachytherapy with hyaluronate injection via paravertebral approach: with DVH comparison to IMRT. Brachytherapy 2013; 12: 8-13.

9. Heinze C, Omari J, Manig M et al. Efficacy and safety of percutaneous computed tomography-guided high-dose-rate interstitial brachytherapy in treatment of oligometastatic lymph node metastases of retroperitoneal space. J Contemp Brachytherapy 2019; 11: 436-442.

10. Kunogi H, Hsu IC, Yamaguchi N et al. CT-guided pelvic lymph nodal brachytherapy. Front Oncol 2021; 10: 532555.

11. Kim Y, Hsu IC, Pouliot J. Measurement of craniocaudal catheter displacement between fractions in computed tomography-based high dose rate brachytherapy of prostate cancer. J Appl Clin Med Phys 2007; 8: 2415.

12. Haviland JS, Owen JR, Dewar JA et al. The UK Standardisation of Breast Radiotherapy (START) trials of radiotherapy hypofractionation for treatment of early breast cancer: 10-year follow-up results of two randomised controlled trials. Lancet Oncol 2013; 14: 1086-1094.

13. NCI. CTCAE. Available from: URL:http://evs.nci.nih.gov/ ftp1/CTCAE/About.html. Accessed May, 17, 2010.

14. Skinner HD, Strom EA, Motwani SB et al. Radiation dose escalation for loco-regional recurrence of breast cancer after mastectomy. Radiat Oncol 2013; 8: 13.

15. Fröhlich G, Mészáros N, Smanykó V et al. Biological dose summation of external beam radiotherapy for the whole breast and image-guided high-dose-rate interstitial brachytherapy boost in early-stage breast cancer. J Contemp Brachytherapy 2020; 12: 462-469. 